\title{
Plans for the LIGO-TAMA joint search for gravitational wave bursts
}

\author{
Patrick J Sutton ${ }^{1}$, Masaki Ando ${ }^{2}$, Patrick Brady ${ }^{3}$, Laura Cadonati ${ }^{4}$, \\ Alessandra Di Credico ${ }^{5}$, Stephen Fairhurst ${ }^{3}$, Lee Samuel Finn ${ }^{6}$, \\ Nobuyuki Kanda ${ }^{7}$, Erik Katsavounidis ${ }^{4}$, Sergey Klimenko ${ }^{8}$, \\ Albert Lazzarini ${ }^{1}$, Szabolcs Marka ${ }^{1}$, John W C McNabb ${ }^{6}$, \\ Saikat Ray Majumder ${ }^{3}$, Peter R Saulson ${ }^{5}$, Hideyuki Tagoshi ${ }^{9}$, \\ Hirotaka Takahashi $^{9,10,11}$, Ryutaro Takahashi ${ }^{12}$, Daisuke Tatsumi ${ }^{12}$, \\ Yoshiki Tsunesada ${ }^{12}$ and S E Whitcomb ${ }^{1}$ \\ ${ }^{1}$ LIGO Laboratory, California Institute of Technology, Pasadena, CA 91125, USA \\ 2 Department of Physics, University of Tokyo, Hongo, Bunkyo-ku, Tokyo 113-0033, Japan \\ ${ }^{3}$ Department of Physics, University of Wisconsin-Milwaukee, Milwaukee, WI 53201, USA \\ ${ }^{4}$ LIGO Laboratory, Massachusetts Institute of Technology, Cambridge, MA 02139, USA \\ 5 Department of Physics, Syracuse University, Syracuse, NY 13244, USA \\ ${ }^{6}$ Center for Gravitational Wave Physics, Department of Physics, The Pennsylvania State \\ University, University Park, PA 16802, USA \\ ${ }^{7}$ Department of Physics, Graduate School of Science, Osaka City University, Sumiyoshi-ku, \\ Osaka, Osaka 558-8585, Japan \\ ${ }^{8}$ Department of Physics, University of Florida, Gainesville, FL 32611, USA \\ ${ }^{9}$ Department of Earth and Space Science, Graduate School of Science, Osaka University, \\ Toyonaka, Osaka 560-0043, Japan \\ ${ }^{10}$ Graduate School of Science and Technology, Niigata University, Niigata, Niigata 950-2181, \\ Japan \\ 11 Yukawa Institute for Theoretical Physics, Kyoto University, Kyoto, Kyoto 606-8502, Japan \\ ${ }^{12}$ National Astronomical Observatory of Japan, Mitaka, Tokyo 181-8588, Japan \\ E-mail: psutton@ligo.caltech.edu
}

Received 19 April 2004

Published 30 September 2004

Online at stacks.iop.org/CQG/21/S1801

doi:10.1088/0264-9381/21/20/023

\begin{abstract}
We describe the plans for a joint search for unmodelled gravitational wave bursts being carried out by the LIGO and TAMA Collaborations using data collected during February-April 2003. We take a conservative approach to detection, requiring candidate gravitational wave bursts to be seen in coincidence by all four interferometers. We focus on some of the complications of performing this coincidence analysis, in particular the effects of the different alignments and noise spectra of the interferometers.
\end{abstract}

PACS number: $04.80 . \mathrm{Nn}$ 


\section{Introduction}

At present several large-scale interferometric gravitational wave detectors are operating or are being commissioned: GEO [1], LIGO [2], TAMA [3] and Virgo [4]. In addition, numerous resonant mass detectors have been operating for a number of years $[5,6]$. Cooperative analyses by multiple observatories could be valuable for making confident detections of gravitational waves and for extracting the most information from them. This is particularly true for gravitational wave bursts (GWBs) from systems such as core-collapse supernovae [8-11], black hole mergers [12, 13] and gamma-ray bursters [14], for which we have limited theoretical knowledge to guide us. Advantages of coincident observations include a decreased rate of false alarms from random noise fluctuations, the ability to locate a source on the sky, the ability to extract polarization information and better statistics on signal parameters [15-17]. Independent observations using different detector hardware and software also decrease the possibility of error or bias.

The TAMA and LIGO Collaborations are currently conducting joint searches for gravitational waves in the LIGO Science Run 2 (S2) and TAMA Data Taking Run 8 (DT8) data, which were collected between 14 February and 14 April 2003 [2, 3]. Three classes of gravitational wave transients are being sought:

(i) unmodelled GWBs from the gamma ray burst event GRB 030329 [18-21];

(ii) inspiral signals from galactic binary neutron star systems (see, for example, [22, 23]); and (iii) unmodelled GWBs without an electromagnetic trigger (see, for example, [24, 25]).

In this paper we report on the planned 'untriggered' search for unmodelled GWBs. This is the first four-interferometer coincidence analysis for $\mathrm{GWBs}^{13}$, involving detectors at three sites. We will focus on some of the challenges facing this joint search, in particular the differences in the noise spectra and in the alignments of the LIGO and TAMA detectors. As we shall see, these motivate a coincidence search between LIGO and TAMA that involves a trade-off between the false alarm rate, the detection efficiency and the bandwidth. The result is a search for high-frequency GWBs (in the range [700-2000] Hz) characterized by a low false alarm rate. We adopt this conservative approach to detection by the LIGO-TAMA detectors in order to gain experience sharing data and performing combined analyses. This search could form a prototype for more comprehensive collaborative analyses in the future.

In section 2 we review the procedure used in the joint search for GWBs. In section 3 we examine some of the challenges facing the joint analysis and their implications. In section 4 we conclude with a few brief comments.

\section{Untriggered bursts analysis}

The LIGO-TAMA joint search for GWBs is structured in the style of the LIGO S1 GWB analysis [24] and the TAMA DT8 GWB analysis [25]. Our goal is to detect GWBs or place upper limits on the rate of GWBs detectable by our instruments. Since we aim to be sensitive to the widest variety of waveforms which contain significant energy in the frequency range of our detectors, we choose to make minimal assumptions about the GWB waveforms; in particular we do not use templates or matched filters. Instead, a variety of non-template based burst detection algorithms are employed to locate candidate GWBs [25-29].

The analysis procedure is straightforward:

(i) Each collaboration independently generates lists of candidate GWBs. These events are characterized by (at minimum) a start and/or 'peak' time, a duration and an amplitude or

${ }^{13}$ See [5-7] for GWB searches involving networks of resonant mass detectors. 
SNR. The LIGO events are also characterized by a frequency range, whereas the TAMA bursts detection scheme uses a fixed set of frequencies between 700 and $2000 \mathrm{~Hz}$ [25]. The event generation procedure may include vetoes of candidate events based on auxiliary data, such as from environmental monitors. In addition, events which are in coincidence in the three LIGO interferometers are subjected to a coherence test, in which the crosscorrelation of the data from each pair of LIGO interferometers is required to exceed a certain threshold [30]. (Further study is required before including TAMA events in this coherence test due to the different alignment of TAMA; see section 3.2.)

(ii) The event lists from the four detectors are compared and candidate GWBs are selected by requiring coincident detection in each interferometer (LIGO and TAMA).

(iii) The rate of accidental coincidences due to the background noise is estimated by repeating the coincidence and coherence tests after artificially shifting the time stamps of one or more lists of events. It is intended that the event generation thresholds in step (i) will be kept high enough so that the average background rate will be less than one event over the observation time (approximately $250 \mathrm{~h}$ ).

(iv) An excess of coincident events above the background will be investigated as a possible detection; in the absence of an excess an upper limit on the rate of detectable events will be set using the background estimate.

(v) The efficiency of the LIGO-TAMA network at detecting real GWBs will be estimated by adding various simulated signals (astrophysical and ad hoc) to the data in Monte Carlo experiments.

It should be noted that the LIGO collaboration is also performing a separate coincidence search for GWBs using the LIGO S2 data alone below $1.1 \mathrm{kHz}$. Candidate GWBs from the two analyses (if any) will be compared.

\section{Challenges for LIGO-TAMA}

A coincidence analysis involving LIGO and TAMA is more complicated than, for example, a coincidence analysis involving the LIGO detectors only. The main complications are that the LIGO and TAMA detectors have different noise spectra and different alignments.

\subsection{Sensitivity versus frequency}

Figure 1 shows representative noise spectra from the LIGO Hanford $4 \mathrm{~km}$ detector and the TAMA detector during S2/DT8 (the other LIGO detectors are similar to the one shown). The individual detectors have maximum sensitivity at very different frequencies-about $250 \mathrm{~Hz}$ for LIGO and $1300 \mathrm{~Hz}$ for TAMA.

If we ignore differences in alignment, then requiring four-fold coincident detection means that the overall sensitivity of the network is limited by the least sensitive detector. This is because our coincident analysis requires a signal to be detected by all interferometers; hence, we choose to focus our search on the minimum of the noise envelope, which occurs around $1000 \mathrm{~Hz}$. Specifically, we require that the frequency range of LIGO events overlaps $[700,2000] \mathrm{Hz}$; this is also the frequency range analysed in the production of TAMA events. Restricting the frequency range will reduce the rate of false alarms due to coincident noise fluctuations, while preserving sensitivity to real GWBs that are detectable by both LIGO and TAMA.

Note that any low-frequency GWBs which are detectable by LIGO but not TAMA will be found in the LIGO collaboration's separate low-frequency search. Thus, there is no danger of 


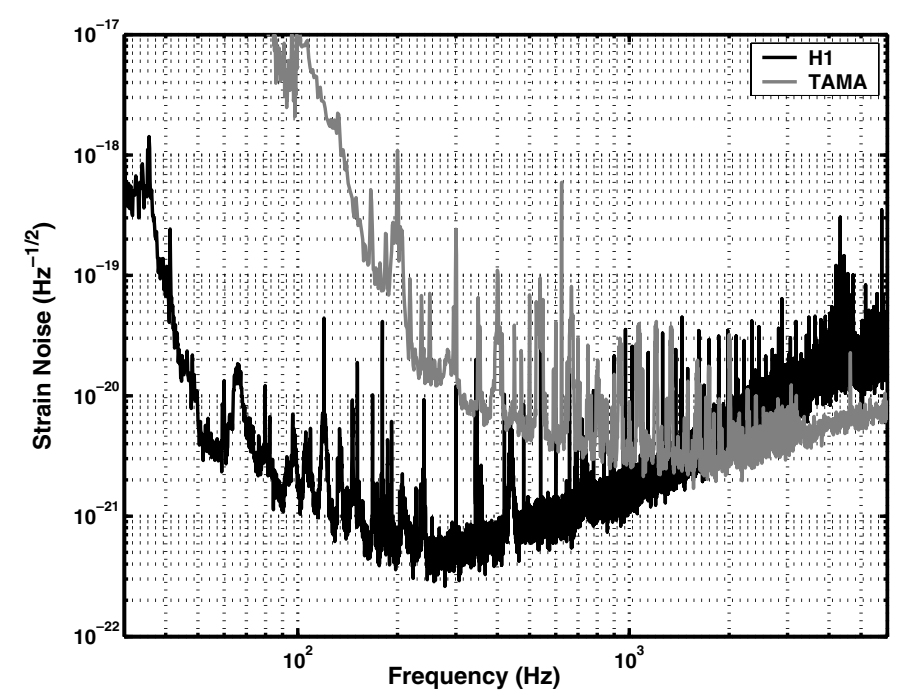

Figure 1. Representative amplitude noise spectra for the LIGO Hanford $4 \mathrm{~km}(\mathrm{H} 1)$ and TAMA detectors. Events from each detector are used in this analysis if they intersect the frequency range [700-2000] Hz, where each interferometer has approximately the same noise level.

a detectable low-frequency GWB being missed due to our choice of frequency range for the LIGO-TAMA coincidence.

\subsection{Antenna patterns}

Figure 2 shows the antenna patterns

$$
\rho \equiv F_{+}^{2}+F_{\times}^{2}
$$

for the LIGO Hanford and TAMA detectors. (The LIGO Livingston antenna pattern is very similar to that of the LIGO Hanford detectors.) These detectors are strongly non-aligned in that the areas of maximum sensitivity for LIGO overlap minima of TAMA sensitivity, and vice versa. That is, LIGO and TAMA look with best sensitivity at different parts of the sky. This complicates LIGO-TAMA coincidence; if we ignore differences in noise levels, then requiring four-fold coincident detection means that the overall sensitivity of the network to a given point on the sky is limited by the minimum of the antenna patterns.

The impact of this alignment difference on the network sensitivity can be estimated via a simple Monte Carlo experiment. Let us assume each interferometer has the same sensitivity for some fiducial signal of interest. We model this detection efficiency as a simple sigmoid in the logarithm of the signal amplitude, as shown in figure $3 .{ }^{14}$ We then generate linearly polarized signals of random amplitude and polarization angle, distributed isotropically across the sky. We project each signal onto the antenna pattern of each instrument, and compute the coincident detection efficiency for different combinations of interferometers ${ }^{15}$. Figure 3 shows the resulting efficiencies as a function of the intrinsic signal amplitude. For example,

${ }^{14}$ We use equation (5.10) of [24] with $a=0.1, b=0$. Choosing $b=0$ is the case in which all four detectors have the same sensitivity; this is approximately true around $1 \mathrm{kHz}$ (see figure 1), and it is the simplest choice for illustrating the effect of the alignment separately from differences in the noise levels. Sigmoid widths of order from $a \simeq 0.07$ to $a \simeq 0.15$ are typical of the values found in the LIGO S1 analysis [24] and in the TAMA DT8 analysis. The network sensitivities depend only weakly on $a$.

${ }^{15}$ See [31] for the efficiency of an alternative network detection algorithm. 

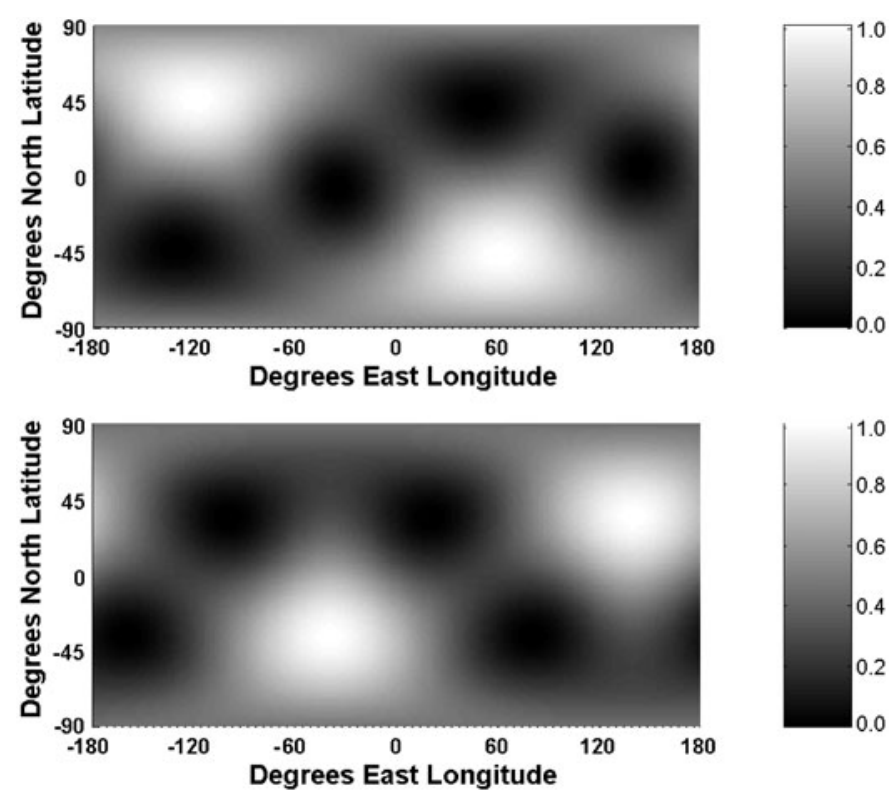

Figure 2. Antenna patterns $\rho$ (1) in Earth-based coordinates (see, for example, [32]). The upper plot is for the LIGO Hanford detectors, while the lower plot is for TAMA. Light areas denote sky directions of the highest sensitivity. The directions of LIGO's maximum sensitivity lie close to areas of TAMA's worst sensitivity and vice versa.

the minimum signal amplitude required for the three (nearly co-aligned) LIGO detectors to have a $50 \%$ chance of simultaneous detection is approximately $4.7 / 2.9 \simeq 1.6$ times larger than the amplitude for $50 \%$ detection by a single interferometer (exemplified here by TAMA). The minimum amplitude for simultaneous detection by both LIGO and TAMA is approximately 2.6 times the single-detector amplitude, or 1.6 times the LIGO amplitude.

The network efficiency is a function of the type of signal studied, and hence figure 3 is only illustrative of what is expected for LIGO-TAMA. Nevertheless, the loss in efficiency when adding detectors to the network is generic when requiring coincident detection. Either collaboration could achieve better sky-averaged sensitivity by not requiring coincidence with the other. However, the loss in efficiency is accompanied by a drop in the false alarm rate. For example, typical false-alarm rates at the single-interferometer level in this analysis are in the range $0.1-1 \mathrm{~Hz}$. Assuming a LIGO-TAMA coincidence window of order $0.1 \mathrm{~s}$, each additional detector added to the network will lower the coincident false alarm rate by a factor of 10-100. This false alarm reduction brings with it improved sensitivity to regions of the sky to which both LIGO and TAMA are simultaneously sensitive and is the principal scientific justification for our joint $\operatorname{search}^{16}$.

Another consequence of the difference in LIGO and TAMA alignments is that the detectors are sensitive to different polarization combinations of a gravitational wave. This effect could be particularly important when cross-correlating events between LIGO and TAMA. Coherence tests have been shown to be a powerful means of reducing coincidence false alarm rates in

${ }^{16}$ An alternative approach would be to lower the thresholds for event generation to keep the network false alarm rate constant as detectors are added to the network. Whether the resulting gain in efficiency would be enough to offset the loss from adding differently aligned detectors depends on the relationship between the false alarm rate and efficiency for each detector. 


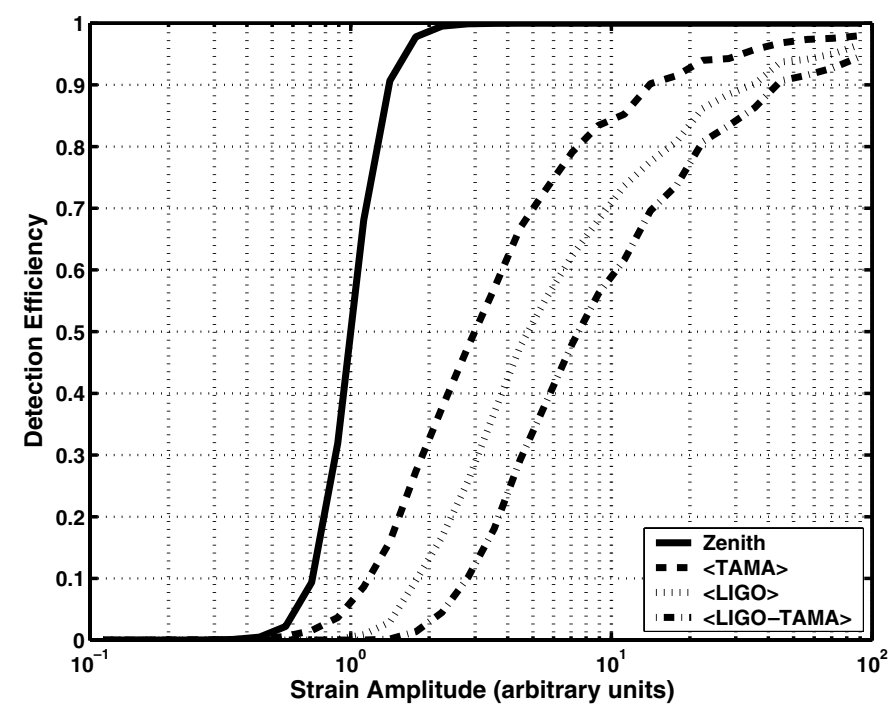

Figure 3. Sample efficiency estimates for the LIGO-TAMA network from Monte Carlo simulations. Each interferometer is assumed to have identical sensitivity to a fiducial source (represented by the zenith curve) when that source is optimally positioned and oriented with respect to the interferometer. The other curves show the efficiency to a population of such sources isotropically distributed over the sky and with random polarization. The TAMA curve shows the detection efficiency of the TAMA detector alone, the LIGO curve shows the efficiency for coincident detection by all three LIGO detectors and the LIGO-TAMA curve shows the efficiency for coincidence detection by TAMA and all three LIGO interferometers.

LIGO [30], and extending these tests between LIGO and TAMA could reduce the network false alarm rate further. This issue is under study.

\section{Summary}

The LIGO and TAMA collaborations are conducting joint analyses of the LIGO S2-TAMA DT8 data collected during February-April 2003. There are three analyses focusing on gravitational wave transients: unmodelled GWBs from GRB 030329, inspiral signals from galactic neutron star binaries and an untriggered search for unmodelled GWBs.

We have reviewed the plans for the untriggered search for GWBs and some of the complications of comparing LIGO and TAMA events. We take a conservative approach to detection, requiring candidate GWBs to be seen in coincidence by all four interferometers at frequencies where all four have comparable sensitivity. While this is expected to result in lower sensitivity of the network compared to the individual interferometers, it is the most conservative approach with respect to false alarms.

\section{Acknowledgment}

The LIGO Laboratory operates under cooperative agreement with the National Science Foundation, grant PHY-0107417. 


\section{References}

[1] Willke B et al 2004 Class. Quantum Grav. 21 S417

[2] Sigg D 2004 Class. Quantum Grav. 21 S409

[3] Takahashi R 2004 Class. Quantum Grav. 21 S403

[4] Acernese F et al 2004 Class. Quantum Grav. 21 S385

[5] Allen Z A et al 2000 Phys. Rev. Lett. 855046

[6] Astone P et al 2003 Phys. Rev. D 68022001

[7] Amaldi E et al 1989 Astron. Astrophys. 216325

[8] Zwerger T and Muller E 1997 Astron. Astrophys. 320209

[9] Dimmelmeier H, Font J and Muller E 2002 Astron. Astrophys. 388917

[10] Dimmelmeier H, Font J and Muller E 2002 Astron. Astrophys. 393523

[11] Ott C, Burrows A, Livne E and Walder R 2004 Astrophys. J. 600834

[12] Flanagan E and Hughes S 1998 Phys. Rev. D 574535

[13] Flanagan E and Hughes S 1998 Phys. Rev. D 574566

[14] Mészáros P 2002 Ann. Rev. Astron. Astrophys. 40137

[15] Finn L S 2001 Phys. Rev. D 63102001

[16] Pai A, Dhurandhar S and Bose S 2001 Phys. Rev. D 64042004

[17] Arnaud N et al 2003 Phys. Rev. D 68102001

[18] Mészáros P 2003 Nature 423809

[19] Price P A et al 2003 Nature $\mathbf{4 2 3} 844$

[20] Hjorth J et al 2003 Nature 423847

[21] Mohanty S D, Marka S, Rahkola R, Mukherjee S, Leonor I, Frey R, Cannizzo J and Camp J 2004 Class. Quantum Grav. $21 \mathrm{~S} 765$

[22] Abbott B et al 2004 Analysis of LIGO data for gravitational waves from binary neutron stars Phys. Rev. D 69 122001 (Preprint gr-qc/0308069)

[23] Takahashi H and Tagoshi H 2004 Class. Quantum Grav. 21 S769

[24] Abbott B et al 2004 Phys. Rev. D 69102001

[25] Ando M et al 2004 Class. Quantum Grav. 21 S1679

[26] Sylvestre J 2002 Phys. Rev. D 66102004

[27] Anderson W et al 2001 Phys. Rev. D 63042003

[28] Klimenko S et al 2004 Class. Quantum Grav. 21 S1685

[29] McNabb J et al 2004 Class. Quantum Grav. 21 S1705 (Preprint gr-qc/0404123)

[30] Cadonati L 2004 Class. Quantum Grav. 21 S1695

[31] Kanda N 2003 Class. Quantum Grav. 20 S761

[32] Althouse W et al 2001 Rev. Sci. Instrum. 723086 